\title{
Characterization of hematopoietic precursor cell Flt3R/ CD164 expression and capacity of colonization formation from different human hematopoietic tissues
}

\author{
Hsiao-Nan Hao ${ }^{1,2}$, Yongtai Zhang ${ }^{2}$, Jane Zhao ${ }^{1}$, Guotong Xu ${ }^{3}$, Yanyun Zhang ${ }^{3}$ \\ ${ }^{1}$ Wayne State University, School of Medicine, ${ }^{2}$ Yangtze Delta Region Institute of Tsinghua University, Zhejiang, ${ }^{3}$ Health Research \\ Institute of Jiaotong University
}

Hematopoietic progenitor cells (HPC) are capable of self-renewal, of differentiation into many different lineages, and have the potential to treat malignant and non-malignant diseases. The cytokine tyrosine kinase receptor fit3 is expressed, and plays a critical role in the early human hematopoiesis such as stem cell self-renewal, ex vivo expansion, differentiation, and maturation. Most of the CD34+ cells at early stage (G0) have capacity to express flt3 receptor (flt3R), however, the expression of flt3R among those cells remains at lower level compared with that of G1 HPC. Many reported results suggest that only highly expressed flt3R cells are able to give rise to colonyforming unit-granulocyte/macrophage (CFU-GM) whereas filt3R-dimmer cells give rise to erythroid progenitor cell-derived colonies (BFU-E). To estimate the HPC restricted erythroid differentiation potential, we examined the flt3R expression and colony formation capacity of HPC from different human hematopoietic tissues including fetal human liver (FL), cord blood (CB), bone marrow tissue (BM) as well as mobilized peripheral blood (MPB). Using the single-sorted CD34+ cells from different hematopoietic tissues (FL, CB, BM and PB) to assess the relationship between immunophenotype expression and functional characteristics, we analyzed both flt3 $\mathrm{R}$ expression and colony formation in HPC from different hematopoietic tissue. Based on the fit3R expression, CD34+ cells could be split into CD34+/flt3R++ and CD34+/flt3R \pm (dimmed) groups. The percentages of each group with flt3R higher and dimmer expression were $11 \pm 4 \%$ vs. $82 \pm 8 \%$ in $\mathrm{FL}, 53 \pm 12 \%$ vs. $41 \pm 4 \%$ in $\mathrm{CB}, 74 \pm 10 \%$ vs. $23 \pm 7 \%$ in $\mathrm{BM}$ and $\mathbf{7 8} \pm \mathbf{1 4 \%}$ vs. $16 \pm 5 \%$ in MPB. Using three-color analysis and coexpression of CD34+/flt3R/Cd164, CD34+/flt3R \pm (dimmed) HPC group isolated from all the tissue samples were CD164 positive. To further analyze the capacity of colony formation from these cells, the percentages of colonies with BFU-E/CFU-GM were $61 \pm 8 \%$ vs. $27 \pm 6 \%$ in FL, $43 \pm 12 \%$ vs. $32 \pm 9 \%$ in $C B, 21 \pm 4 \%$ vs. $26 \pm 6 \%$ in $B M$ and $16 \pm 5 \%$ vs. $23 \pm 7 \%$ in MPB. In addition, CFU-GM colonies were dramatically reduced using flt3R antisense oligonucleotides (ODN) to treat cells with higher fit3R expression. However, we did not find the number of BFU-E colony increased with the same treatment. These data demonstrate that the flt3R expression alteration associates with cell native maturation and monocytic differentiation despite lack of understanding of the complete flt3R expression mechanisms regardless HPC cell resource. Decrease of the fit3R expression reduced the CFU-GM formation but did not affect BFU-E. Taken all together, our data also indicate that HPC from FL appear to represent the best quality cells to give rise to erthroid products compared with other HPC from CB, BM and MPB tissue.

Keywords: hematopoietic progenitor cells, flt3 receptor, CD164

Cell Research (2008) 18:s117. doi: 10.1038/cr.2008.207; published online 4 August 2008

Correspondence: Hsiao-Nan Hao

E-mail: hsiao-nan.hao@wayne.edu

Dr Hsiao-Nan Hao, graduated from Qingdao Medical College and Brown University, and received his post-doctoral training from University of Michigan and Albert Einstein College of Medicine. He had been awarded a NIH Fellowship and trained in pathology before he was recruited as an assistant professor in Wayne State University 1997. His on going research interests include fetal human stem cell study (purification/ differentiation/transplantation) and antisense ODN gene regulatory studies (anti-inflammation therapy). Using of fetal human stem cells, he have demonstrated the neural transdifferentiation capacity of human hematopoietic stem cells and vulnerability of neural stem cell to posing exposure in vitro. His stem cell research is much related to pre-clinic hematopoietic and neural transplantation.Hsiao-nan.hao@med.wayne.edu 